Irish Math. Soc. Bulletin

Number 76, Winter 2015, 27-28

ISSN 0791-5578

\title{
MATHEMATICAL MODELS OF SEASONALLY MIGRATING POPULATIONS
}

\author{
JOHN G. DONOHUE
}

This is an abstract of the $\mathrm{PhD}$ thesis Mathematical models of seasonally migrating populations written by J. Donohue under the supervision of Dr. P. T. Piiroinen at the School of Mathematics, Statistics, and Applied Mathematics, National University of Ireland, Galway and submitted in September 2015.

The phenomenon of seasonal migration has attracted a wealth of attention from biologists. However, the dynamics of migratory populations have been little considered. In this thesis, we use differential equations to model the variation in abundance of seasonally migrating populations.

Our contribution to the field begins with a representation of seasonal breeding. We use piecewise-smooth differential equations to model the variation in the size of a population that has a short interval each year during which successful reproduction is possible. We first consider a one-species model which illustrates the dynamics of a population of specialist feeders over the course of a single breeding season and use it to examine how reproductive success depends on the population's distribution of breeding dates. We then introduce time-dependent switches to extend the model to a broader class of species. This allows us to consider the effect of climate change on populations that annually travel long distances.

We then shift focus to consider interactions between migrants and species at higher levels in the food web. Predatory pressure influences almost all populations to some extent. Here, however, interactions may occur for just a brief period each year before the populations involved become spatially separated. The range of a migrating population may overlap with that of a population of predators for a

2010 Mathematics Subject Classification. 92D25, 34C25, 37G15.

Key words and phrases. dynamics, populations, seasonality, periodic.

Received on 16-12-2015.

Support from Irish Research Council through the Embark Initiative is gratefully acknowledged. 
single season. We outline a framework for examining how this kind of "transient" predation influences the dynamics of the prey population. We are then able to examine how a migratory population may be overwhelmed by the fleeting influence of members of other species.

Finally, as an alternative to the aforementioned models, we outline a different approach to modelling migration, namely using partial differential equations instead of ordinary differential equations. In this way, we provide two distinct templates for the future exploration of the dynamical features of such populations.

(from October 2015) MACSI, UNIVERSITY OF LIMERICK

E-mail address, J. Donohue: john.donohue.nui@gmail.com 